\title{
Clinical evaluation of motion and position sense in the upper extremities of the elderly using motion analysis system
}

This article was published in the following Dove Press journal:

Clinical Interventions in Aging

16 July 2014

Number of times this article has been viewed

\author{
Kuan-yi $\mathrm{Li}^{\prime}$ \\ Yi-hui Wu \\ 'Department of Occupational Therapy \\ and Graduate Institute of Behavioral \\ Sciences, Healthy Aging Research \\ Center, Chang Gung University, \\ Kwei-shan, Tao-Yuan, Taiwan; \\ ${ }^{2}$ Department of Rehabilitation, \\ Taoyuan Chang Gung Memorial \\ Hospital, Kwei-shan, Tao-Yuan, Taiwan
}

Correspondence: Kuan-yi Li Department of Occupational Therapy and Graduate Institute of Behavioral Sciences, Healthy Aging Research Center, Chang Gung University, 259 Wen-hwa Ist Road, Kwei-shan, Tao-yuan, Taiwan, 333

Tel +886 321 I 8800 ext 3676

Fax +88632118421

Email kyli@mail.cgu.edu.tw

\begin{abstract}
The purpose of this study was to measure kinesthetic accuracy in healthy older adults by using arm position and motion matching tests. We investigated the effect of task type, joint angle, and matching arm results on kinesthetic accuracy in the upper extremities of 17 healthy right-handed older adults. Blinded subjects were asked to match positions and motions at four reference joint angles: 1) shoulder flexion, $0^{\circ}-60^{\circ}$; 2) elbow flexion, $90^{\circ}-135^{\circ}$; 3) wrist extension, $0^{\circ}-50^{\circ}$ in the sagittal plane; and 4) shoulder abduction, $0^{\circ}-60^{\circ}$ in the frontal plane. The absolute difference in angular displacement between the reference and matching arms was calculated to determine kinesthetic accuracy. Results showed that subjects were more accurate at matching motion than position tasks $(P=0.03)$. Shoulder and elbow joints were more sensitive than wrist joints in perceiving passive positions and motions $(P<0.05)$. The effect of the matching arm was found only when matching the joint angles of shoulder abduction and wrist extension $(P<0.01)$. These results are comparable to findings of other studies that used machine-generated kinesthetic stimuli. The manual measurement of kinesthetic accuracy could be effective as a preliminary screening tool for therapists in clinical settings.
\end{abstract}

Keywords: joint position matching, proprioception, velocity perception

\section{Introduction}

Proprioception has been defined as awareness of and ability to sense the position of limbs, trunk (position sense), and kinesthesia as the awareness of motion of the human body (motion sense). ${ }^{1}$ Awareness and ability are essential for optimal muscular control, coordination, and stability when planning movements for the performance of daily activities. ${ }^{2-5}$

In a rehabilitation setting, therapists often rely on proprioceptive and kinesthetic accuracy to determine intervention programs and evaluate treatment effectiveness. ${ }^{6} \mathrm{~A}$ comprehensive sensory evaluation can provide useful information for determining clinical treatment. However, results from Lincoln et al showed that inter-rater reliability of sensory assessment for stroke survivors was poor due to variability between assessors and subjects. ${ }^{7}$ A reliable and standardized assessment is needed to increase the reliability of assessment and minimize physical and mental demands on subjects. Although the validity and reliability of the arm matching test has been little researched, ${ }^{8}$ the magnitude of end-position errors has been thought to be a good indicator of acuity for motion and position sense. ${ }^{9}$

The arm position matching test, a common proprioceptive measurement, is used to measure the ability of a subject to perceive limb position by moving a single joint on one side and matching its exact position on the other. ${ }^{10-12}$ During the test, subjects are required to move the testing joint to match the reference joint at the desired position. 
At the end of each trial, examiners visually determine proprioceptive accuracy. Using an ordinal scale, accuracy is noted as absent (no appreciation), impaired (inaccurate detection), or intact (precise awareness). ${ }^{6,13}$

Although previous studies have reported on the accuracy of position and motion sense for various populations, ${ }^{14-22}$ the experimental paradigms used in those studies are difficult for clinical practitioners to apply on patients due to the following reasons. First, researchers used their own customized apparatuses to measure accuracy of position and motion sense because uniform commercial apparatuses were unavailable. Second, lack of a standardized test protocol made it difficult to compare results across studies. Finally, some experimental paradigms involved the use of memory issues, applications that were impractical when patients suffered from central nervous system (CNS) impairments.

As a result, the current study examined the most widely used clinical position and motion sense assessments, the arm position and motion matching tests, to investigate their suitability for screening proprioceptive and kinesthetic ability in a clinical setting. When performing the tests during assessments, therapists did not move the subject's reference arm back to the starting position. Furthermore, subjects were not required to memorize arm positions or motions during the tests; therefore, memory issues were excluded. The tests were simple and easy to administer to patients in a clinical setting. This study investigated whether the arm position matching test could be a useful screening test for measuring passive position sense of the upper limbs by manually imposing passive movements on the upper extremities. Subjects were required to mirror the position while blinded to the tests.

This study measured proprioceptive and kinesthetic accuracy in healthy older adults by using the arm matching position and motion tests. We investigated the effects of task types (position matching vs motion matching), joint angles (shoulder flexion $0^{\circ}-60^{\circ}$, shoulder abduction $0^{\circ}-60^{\circ}$, elbow flexion $90^{\circ}-135^{\circ}$, and wrist extension $0^{\circ}-50^{\circ}$ ), and matching arm (preferred vs non-preferred hand) on the accuracy of position and motion sense of the joints in the upper extremities. The main purpose of this study was to investigate whether arm matching position and motion tests can provide clinical results compatible with those of previous studies that used a custom-made apparatus as an initial screening tool.

\section{Methods}

\section{Subjects}

Seventeen healthy right-handed older adults $(7$ males, 10 females) participated in this study. Mean age was $62.59 \pm 4.60$ years (mean $\pm \mathrm{SD}$ ) and ranges between 56-70 years. Mini Mental State Examination (MMSE) results were $28.65 \pm 1.00$ (mean $\pm \mathrm{SD}$ ). All subjects signed an informed consent form approved by the Institutional Review Board of Chang Gung Memorial Hospital. Inclusion criteria were: 1) Right-handedness. Only right-handed subjects were included according to the Edinburgh Handedness Inventory; ${ }^{23} \mathrm{~b}$ ) No cognitive impairment. Subjects with a score $<24$ on the MMSE were excluded; ${ }^{24}$ and c) No presence of neurological disease such as stroke or diabetes that could interfere with kinesthesia. A medical history interview was performed to exclude subjects with past severe arm injuries.

\section{Kinematic measurements}

We used a seven-camera Vicon MX motion analysis system (Oxford Metrics Inc., Oxford, UK) with a sampling rate of $120 \mathrm{~Hz}$ to capture the movement of subjects during arm position and motion matching tests. A total of nine spherical markers each with a diameter of $5 \mathrm{~mm}$ were used (four markers on each arm and one on the sternum). Bony landmarks were the sternum, acromion process, lateral epicondyle, styloid process of the ulna, and dorsal surface of the third metacarpophalangeal joint. Similar marker placements have been used in previous studies. ${ }^{25-27}$ Prior to data collection, the system was reconfigured, calibrated, and test data were collected. This initial process required approximately $40 \mathrm{~min}-$ utes. For each individual testing session, the experimenters spent 10 to 15 minutes preparing the system for testing.

Shoulder flexion was determined by the angle between the vector joining the ipsilateral acromion-lateral epicondyle markers and the vector from the acromion process towards the hip in the sagittal plane. Shoulder abduction was calculated using the angle between the vector joining the ipsilateral acromion-lateral epicondyle markers and the vector from the acromion process towards the hip in the frontal plane. Elbow flexion was determined by the angle between the vector formed by the ipsilateral acromion-lateral epicondyle markers and a vector defined by the lateral epicondyle and styloid process of the ulna in the sagittal plane. Wrist extension was determined by the angle between the vectors formed by the ipsilateral lateral epicondyle and styloid process of the ulna and a vector defined by the styloid process of the ulna and third metacarpophalangeal joint in the sagittal plane.

\section{Procedure}

Due to a lack of standardized testing protocols for clinics, we based the evaluation procedure on previous research and current clinical practice. ${ }^{6,14,28}$ Measuring accuracy of position and motion sense in the upper extremities normally includes the 
major joints, and excludes the end position, which can generate extra cues other than proprioceptive information. ${ }^{8,29}$ In general, the evaluation was performed from the proximal to the distal joint, and was simple for clinical practitioners to administer. Four joint angles were selected for the arm position and motion matching tests: 1) shoulder flexion (SF), $0^{\circ}-60^{\circ} ; 2$ ) elbow flexion (EF), $\left.90^{\circ}-135^{\circ} ; 3\right)$ wrist extension (WE), $0^{\circ}-50^{\circ}$ in the sagittal plane; and 4) shoulder abduction (SA), $0^{\circ}-60^{\circ}$ in the frontal plane. A detailed graphical representation of the joint angles is shown in Figure 1.

Each subject was seated in a chair adjusted to the subject's height. The subject's forearm rested with an elbow flexion of $90^{\circ}$ for the starting position. Vision was then blocked by opaque glasses, to ensure that the subject relied exclusively on kinesthesia to perform the required tasks. Each subject visited the laboratory once and both arms were tested. Each joint angle was measured three times, and one joint was moved each time. Before data recording, practice trials were performed without the use of opaque glasses to familiarize subjects with the experimental tasks. To ensure consistency of angular displacement imposed by the experimenter, three goniometers were placed on the table and the back of the subject's chair so that the experimenter could visualize the desired reference joint angles to enhance accuracy. The experimental setup is shown in Figure 2.

The order of the experiment was balanced by a Latin square design to prevent a potential order effect. Therefore, each subject was randomly assigned to the following four blocks: 1) position matching task with the non-preferred side as the matching arm; 2) position matching task with
A



C

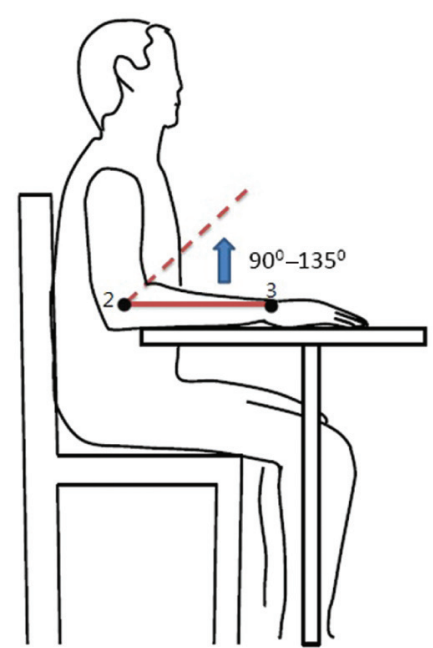

B

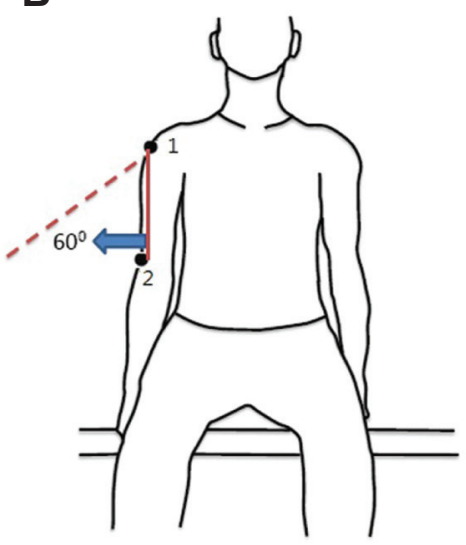

D

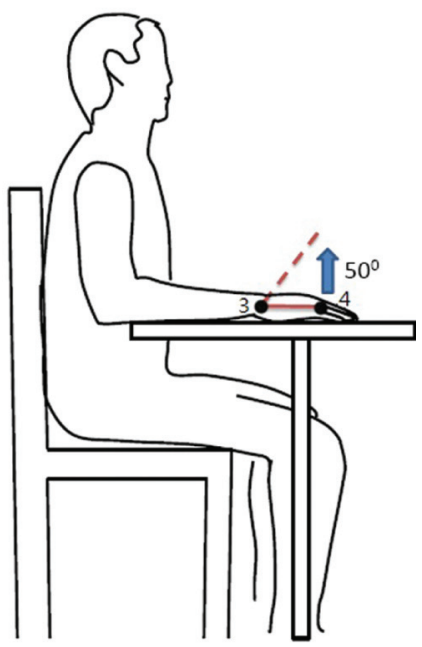

Figure I Tested joint angles and marker placement.

Notes: (A) shoulder flexion $0^{\circ}-60^{\circ}$ in the sagittal plane; (B) shoulder abduction $0^{\circ}-60^{\circ}$ in the frontal plane; (C) elbow flexion $90^{\circ}-135^{\circ}$ in the sagittal plane; and (D) wrist extension $0^{\circ}-50^{\circ}$ in the sagittal plane. Spherical markers (illustrated here with black numbered spheres) were attached to the following bony landmarks for calculations of joint angles: (I) the acromion process; (2) the lateral epicondyle of the elbow; (3) the styloid process of the ulna; and (4) third metacarpophalangeal joint. Solid lines represent the starting position and dashed lines represent the desired joint angles. 


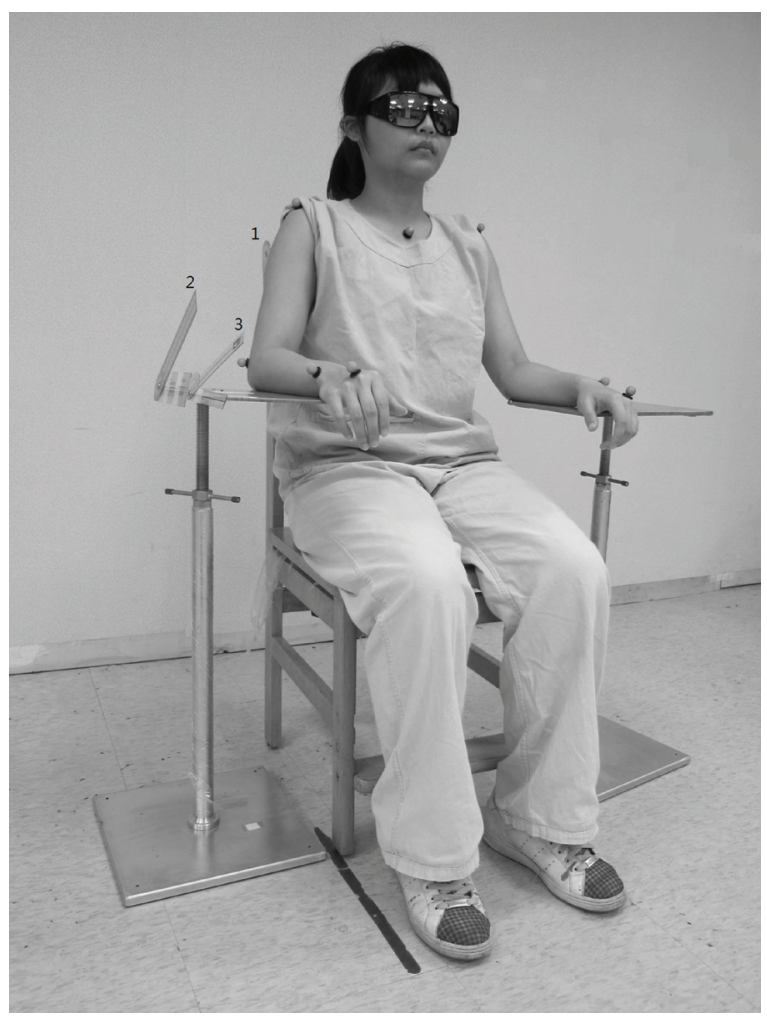

Figure 2 Experimental setup.

Notes: The subject's arms were positioned in a relaxed position and supported by side tables adjusted to each subject's height. Three goniometers (labeled I, 2, 3) were attached to the chair and the side tables for visualization of the desired joint angles and manipulation of the subject's limb to the given joint position.

the preferred side as the matching arm; 3) motion matching task with the non-preferred side as the matching arm; and 4) motion matching task with the preferred side as the matching arm. Each block contained 12 trials; in total 48 trials were conducted, administered by a certified occupational therapist. To ensure the reliability of the imposed displacement and movement, the occupational therapist was trained to follow the experimental protocol and to move the individual joint to the desired angles using the goniometers attached to the chair. To prevent possible extra cues during arm matching tasks, the therapist was instructed to hold the neighboring joints instead of the muscle belly at the desired angles.

In the arm position matching test, the examiner moved the reference arm to the testing joint angles. To mimic the actual measuring procedures used in clinics, the experimenter moved each joint angle for 2 to 4 seconds and maintained the position while subjects moved the matching arm to mirror the joint angle. During the arm motion matching test, the examiner moved the reference arm and asked the subject to concurrently mirror the motion with the matching arm. Subjects were instructed to match the imposed velocity with the matching arm as soon as they were aware of the limb motion. The experimenter isolated the tested joint and attempted to maintain a steady speed for each trial. In both the arm position and motion matching tests, each joint angle measurement was repeated until three correct matching trials were recorded. At the end of each trial, subjects were required to verbally confirm that both joint angles were identical and at symmetrical positions. For the motion sense task, subjects were further required to verbally confirm whether the motion at both joint occurred at the same speed.

\section{Data analyses}

We used a customized algorithm based on MATLAB ${ }^{\circledR}$ (The MathWorks, Inc., Natick, MA, USA) to calculate angular displacement for each trial. All kinematic data were filtered by a low pass Butterworth fourth-order filter with a cut-off frequency of $5 \mathrm{~Hz}$. The outcome measure was the difference in angular displacement (absolute error) between the reference and matching arms. The absolute error was computed in degrees by subtracting the angular displacement of the reference arm from that of the matching arm. As the most widely used measure of proprioceptive and kinesthetic accuracy, the absolute error can distinguish differences among various populations. ${ }^{13-15,30,31}$ An example calculation of absolute error is shown in Figure 3. For position sense tasks, random and constant errors were calculated to show directional information.

A three-way repeated measure analysis of variance (ANOVA) was performed to determine differences in within-subject factors for position and motion sense accuracy followed by post hoc analysis. The three within-subject factors were tasks (position matching vs motion matching),

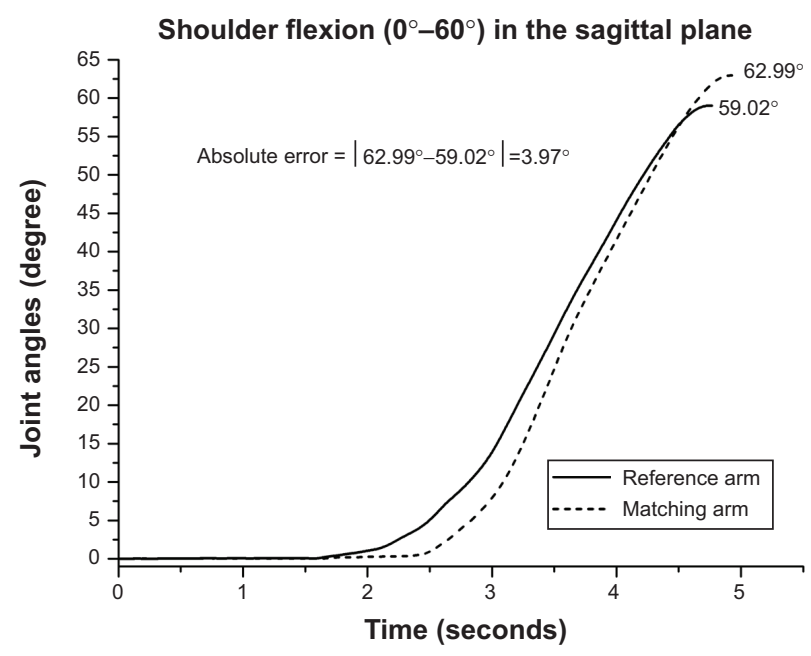

Figure 3 Example calculation of absolute error.

Notes: Shown here is a single trial from one participant. Absolute error was calculated by taking the difference between the final endpoint of the reference arm and the matching arm. 
Table I Absolute error (mean \pm SD) for each condition in degrees

\begin{tabular}{llllll}
\hline Matching arm joint angle & \multicolumn{2}{l}{ Position matching task } & & \multicolumn{2}{l}{ Motion matching task } \\
\cline { 2 - 3 } & Right & Left & & Right & Left \\
\hline SF & $8.14 \pm 5.70$ & $6.60 \pm 5.16$ & $6.98 \pm 5.09$ & $5.63 \pm 3.90$ \\
SA & $10.67 \pm 6.63$ & $8.91 \pm 5.49$ & & $10.59 \pm 8.52$ & $6.54 \pm 4.85$ \\
EF & $6.66 \pm 5.71$ & $7.59 \pm 5.39$ & & $8.76 \pm 6.86$ & \\
WE & $12.58 \pm 7.65$ & $18.16 \pm 11.49$ & & $11.17 \pm 7.88$ & $14.76 \pm 10.41$ \\
\hline
\end{tabular}

Abbreviations: $\mathrm{SF}$, shoulder flexion $0^{\circ}-60^{\circ}$; SA, shoulder abduction $0^{\circ}-60^{\circ}$; EF, elbow flexion $90^{\circ}-135^{\circ}$; WE, wrist extension $0^{\circ}-50^{\circ}$; SD, standard deviation.

joint angles (SF vs SA vs EF vs WE), and matching arms (preferred arm vs non-preferred arm). The significance level was $P<0.05$.

\section{Results}

Because passive position and motion were imposed manually by the experimenter, we examined the intraclass correlation coefficient (ICC) of angular displacement for each joint angle to ensure reliability. The ICC for each joint angle was between 0.6 and 0.8 , which has been defined as an acceptable agreement. ${ }^{32}$ The average velocity of joint angle movement imposed manually by the therapist was $20.24 \pm 3.95(\%)$. The mean absolute error for each joint angle and task are presented in Tables 1 and 2.

The results from the repeat ANOVA did not yield a significant task $\times$ joint angle $\times$ matching arm interaction effect for kinesthetic accuracy $\left(F_{2.27,111.27}=0.69, P=0.52\right)$; however, the joint angle matching arm interaction effect was signifi$\operatorname{cant}\left(F_{2.51,122.97}=8.28, P<0.05\right)$. The simple main effect was further analyzed and a post hoc analysis using least significant difference was performed for a significant effect.

\section{Effect of task type}

The absolute error was $9.91^{\circ} \pm 0.35^{\circ}$ (mean $\pm \mathrm{SD}$ ) for the position matching test and $8.90^{\circ} \pm 0.40^{\circ}$ (mean $\pm \mathrm{SD}$ ) for the motion matching test. A statistically significant difference was found between position and motion matching tests $\left(F_{1,49}=4.82, P=0.03\right)$, and results showed that subjects performed more accurately in the arm motion matching test than in the arm position matching test.

\section{Effect of joint angles}

Further analysis found significant differences in absolute error for joint angles in the preferred $\left(F_{3,147}=8.02, P<0.001\right)$ and non-preferred arms $\left(F_{2.3,112.5}=39.63, P<0.001\right)$. Post hoc analyses showed that subjects were least accurate in matching wrist extension $0^{\circ}-50^{\circ}$ compared to shoulder flexion $0^{\circ}-60^{\circ}$, shoulder abduction $0^{\circ}-60^{\circ}$, and elbow flexion $90^{\circ}-135^{\circ}$ (all $P<0.05$; Figure 4).

\section{Effect of matching arm (using preferred arm vs non-preferred arm)}

Absolute error was $9.45^{\circ} \pm 0.50^{\circ}$ for the preferred arm and $9.37^{\circ} \pm 0.27^{\circ}$ for the non-preferred arm. Further analysis showed that a significant simple main effect was found in shoulder abduction $\left(F_{1,50}=9.55, P=0.003\right)$ and wrist extension $\left(F_{1,50}=8.88, P=0.004\right)$. For shoulder and elbow flexion, no significant difference was found in absolute error for using the preferred or non-preferred arm. However, using the nonpreferred arm to match shoulder abduction $\left(0^{\circ}-60^{\circ}\right)$ and the preferred arm to match wrist extension $\left(0^{\circ}-50^{\circ}\right)$ resulted in greater accuracy than using the other arms.

\section{Discussion}

We examined proprioceptive and kinesthetic accuracy in healthy older adults using arm matching position and motion tests. Based on our results, the subjects demonstrated greater accuracy in the motion matching tasks; wrist joint was less sensitive than proximal joint. The difference between preferred and non-preferred arms was inconsistent among joint angles.

Table 2 Constant error and random error for position sense in degrees

\begin{tabular}{|c|c|c|c|c|}
\hline \multirow{3}{*}{$\begin{array}{l}\text { Matching arm } \\
\text { joint angle }\end{array}$} & \multicolumn{4}{|c|}{ Position matching task } \\
\hline & \multicolumn{2}{|l|}{ Right } & \multicolumn{2}{|l|}{ Left } \\
\hline & Constant error & Random error & Constant error & Random error \\
\hline$\overline{\mathrm{SF}}$ & -0.21 & 9.92 & -1.59 & 8.11 \\
\hline SA & -1.36 & 12.45 & 4.50 & 9.40 \\
\hline EF & -1.90 & 8.92 & -1.42 & 9.18 \\
\hline WE & -11.75 & 9.18 & -11.48 & $|8.5|$ \\
\hline
\end{tabular}

Abbreviations: $\mathrm{SF}$, shoulder flexion $0^{\circ}-60^{\circ}$; SA, shoulder abduction $0^{\circ}-60^{\circ}$; EF, elbow flexion $90^{\circ}-135^{\circ}$; WE, wrist extension $0^{\circ}-50^{\circ}$. 

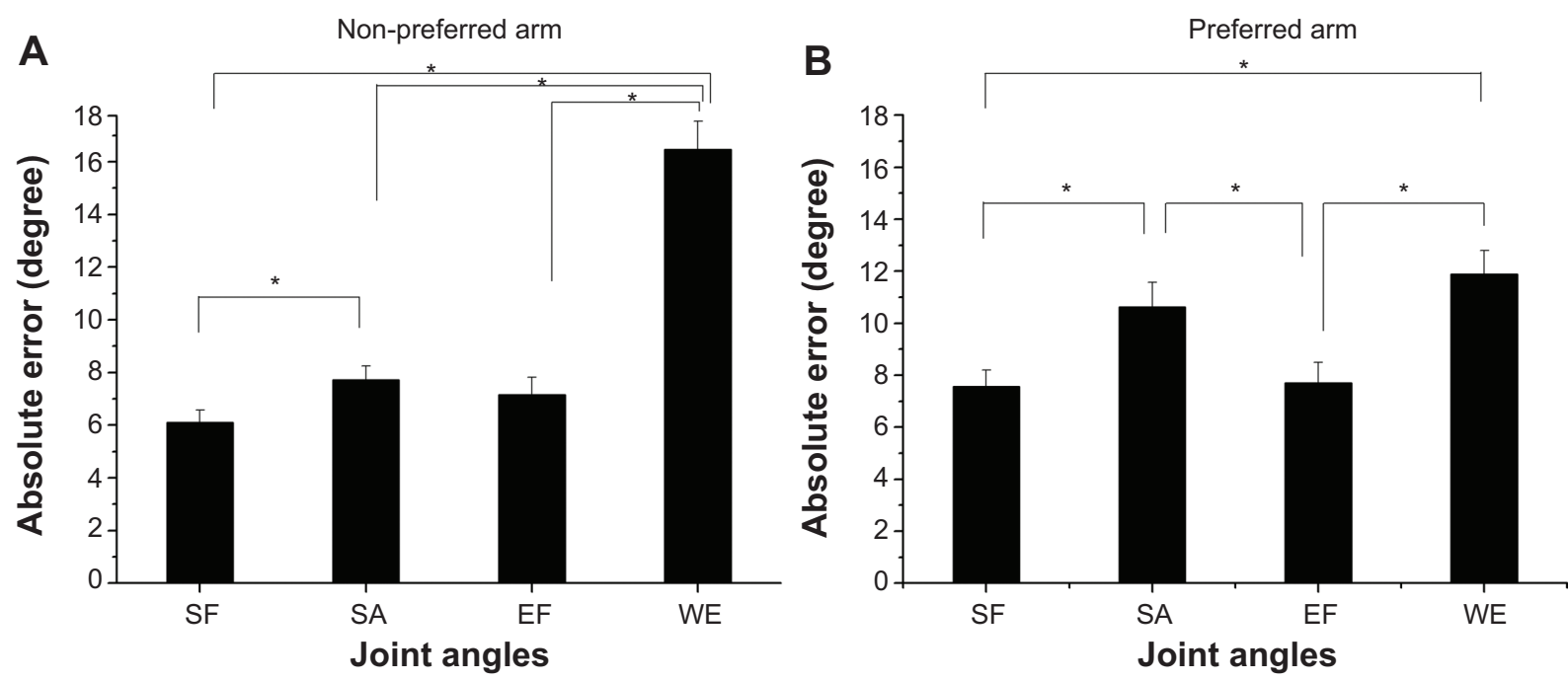

Figure 4 The effect of joint angle on the $(\mathbf{A})$ non-preferred arm and $(\mathbf{B})$ preferred arm. Note: $* P<0.05$.

Abbreviations: $\mathrm{SF}$, shoulder flexion $0^{\circ}-60^{\circ}$; SA, shoulder abduction $0^{\circ}-60^{\circ}$; EF, elbow flexion $90^{\circ}-135^{\circ}$; WE, wrist extension $0^{\circ}-50^{\circ}$.

Current findings indicate that the mean absolute error was reduced in the motion matching test compared to the position matching test, consistent with a previous study. ${ }^{33}$ The discrepancy in proprioceptive accuracy between task types could be due to the proprioceptive information used by each task. However, the method by which proprioceptive information was used during movement has yet been fully understood. ${ }^{34}$ Previous research has shown that the CNS uses all available cues to complete a task. ${ }^{34}$ One possible explanation is that the reference arm provided proprioceptive information to the CNS in both tasks but used it differently in position and motion matching tasks. Several studies have suggested that the right hemisphere is dominant in processing proprioception and kinesthesia and responsible for processing input from muscle spindles, especially in the primary motor area. ${ }^{35,36}$

In the position matching test, the proprioceptive inputs signaled the velocity and end position but subjects did not move the matching arm until the reference arm was placed at the given angle. However, in the motion matching test, the proprioceptive information signals served as "on-line" feedback in providing current status to the CNS to estimate the next state of the limb. By comparing current and predictive forthcoming status, an individual can monitor the performance during the trial and immediately adjust velocity and end position; therefore, proprioceptive accuracy can be elevated in the motion matching test.

Another possible explanation is that position and motion sense are two separate senses, generated from separate processing but having the same receptors. ${ }^{37}$ Evidence from a muscle fatigue study suggests that significantly elevated errors occur only in the elbow position matching test and not in the motion matching test. ${ }^{33}$ In another experiment, blindfolded subjects were required to repeat movements 70 times between two targets. Results showed that the hand position deviated but direction and distance did not. ${ }^{38}$ These findings indicate that position and motion sense can be processed separately, and that position sense is affected more easily by muscle status (fatigue) and nature of the task (repeated movements) than motion sense. As a result, these findings could also explain why subjects in this study performed more accurately in the motion matching task than the position matching task.

Accuracy of the arm matching test at the shoulder and elbow joints was greater than that at the wrist joints in both position and motion matching tests; however, the difference in accuracy between the shoulder and elbow joints remains unclear. This finding concurs with that of Hall and McCloskey who measured the detection threshold of passive movements in shoulder, elbow, and finger joints. ${ }^{28}$ An explanation for this discrepancy is that change in fascicle length per degree was greater in the proximal than distal portion of the muscle. ${ }^{28}$ The joint was considered to be more sensitive in perceiving positions and motions, with more change in the muscle length per degree. Another explanation is that the CNS processed proprioceptive information from multiple joints and integrated interjoint dynamics when performing distal joint movements. ${ }^{5}$ In the current study, we moved only one joint at a time. The group of muscles surrounding the proximal joint also sensed change at the distal portion and provided a stabilizing function. When moving the wrist joint, the subject must stabilize the proximal segment of the limbs to manipulate objects with the hands or fingers. 
Furthermore, results from the computational study of Scott and Loeb showed a descending gradient from proximal to distal joints for position sense accuracy. ${ }^{39}$ The authors proposed that the distribution of muscle spindles around each joint could explain differences in perception of position sense and commented on the relative importance of proximal joints in localizing the endpoint. The effect of gravity and sense of effort were unlikely explanations for the discrepancy in proprioceptive sensitivity, since most joint angles were measured in the sagittal plane under the influence of gravity and we attempted to minimize the influence of gravity to the greatest possible extent with our methodology. Additionally, each joint angle was passively moved by the experimenter, who minimized the sense of effort with the controlled and slow nature of the tested movements. Given these conditions, muscle spindles would be the primary proprioceptor to provide information for all joint angles. Our findings indicate that matching wrist extension $0^{\circ}-50^{\circ}$ was the least accurate joint angle in both position matching and motion matching tasks.

Detailed analyses showed that the difference between the preferred and non-preferred arm was intriguing in the arm position and motion matching tests. Previous research has found the non-preferred arm to be more accurate at performing target-matching tasks than the preferred arm when tasks require both memory and hemisphere transfer. ${ }^{30,40-42}$ Our results did not show systematic limb asymmetry in proprioceptive and kinesthetic accuracy because we employed contralateral matching tasks without reliance on memory. In addition, most studies measured the upper extremity in the elbow and thumb joints; and, we did not have the results from shoulder and wrist joints to compare ${ }^{30,40-42} \mathrm{By}$ contrast, results from vibration studies suggest that proprioceptive asymmetry in upper extremities is dependent on sex, matching hand, and handedness. ${ }^{14}$ For right-handed subjects, studies have found that only males demonstrate significant proprioceptive asymmetry in motion matching tasks. In summary, asymmetric proprioceptive accuracy across limbs was task-dependent and findings were inconsistent and difficult to compare across different experimental protocols.

The accuracy of limb position sense is affected by many factors. First, the issued motor command has been shown to contribute to the ability to sense limb position. ${ }^{43-46}$ Gandevia et al first reported that the level of effort used in generation of the motor command is graded with the amount of position illusion of wrist displacement during hand paralysis ${ }^{43}$ In the current study, subjects actively moved the matching arm in the vertical plane during the position matching test to match the reference arm; therefore, the source of the position signal in the matching arm was a combination of inflow and outflow signals. Second, the effect of muscle history of the reference arm, such as muscle fatigue and conditioning might have generated a position matching error because of the initial status of the muscle spindles. ${ }^{33,45,47}$ Previous studies have found that position matching errors increased when arms are supported by the experimenter, as in the current study; ${ }^{45,48}$ thus, the accuracy of our subjects might have been affected to a greater extent than subjects in other studies using unsupported tasks. Third, the status of the matching arm can influence the position matching error. ${ }^{44,49}$ Previous studies have focused on the effect of the reference arm for position matching tasks. ${ }^{33,43,47}$ However, recent studies have suggested that the condition of the matching arm, such as arm stiffness and conditioning of the muscles, affects the error pattern in the proprioceptive tasks. ${ }^{44,49}$ Finally, the effect of gravity could result in a proprioceptive illusion. ${ }^{50}$ Ansems et al reported that performing position matching tasks with a relaxed arm in the horizontal plane leads to similar results as performing the task in the vertical plane with the arm supported. ${ }^{47}$ Current protocols combined position matching tasks in the horizontal plane with relaxed arms, and in the vertical plane with the arms supported; therefore, the major proprioceptive signal is from the muscle spindles and measurements from the four selected joint angles are comparable. ${ }^{47}$

To ensure the reliability and reproducibility of these results, proper training is required for clinical practitioners to perform the assessment. This is primarily because previous research has shown poor inter-rater reliability for sensory assessment. ${ }^{7}$ Although standardized machinebased assessments have been developed to examine limb position quantitatively and have shown good inter-rater and test-retest reliability in stroke patients, they are not widely available in clinics. ${ }^{19,20}$ Moreover, proprioception has been reported as the main type of somatosensory assessment applied in clinical practice and upper extremities were the most tested body areas. ${ }^{51}$ Therefore, combining training for clinical practitioners and assistance from a standardized device could minimize the measurement bias to obtain reliable results for clinical practice.

\section{Conclusion}

To the best of our knowledge, this is the first study to examine the suitability of using arm matching position and motion tests performed manually by a therapist. Because these tests are commonly used to measure position and motion sense accuracy in clinics, establishing standardized protocols is necessary for evidence-based practice. The results of this 
study are comparable to those of previous studies that used machine-generated kinesthetic stimuli. The manual measurement of accuracy of position and motion sense in clinical settings can be an effective screening tool; however, therapists must be properly trained. Although previous studies have examined detection thresholds for the upper extremities, they used customized machines to provide consistent stimuli. Most studies measured only the accuracy in perceived elbow angular displacement in the transverse plane. The machines, which are expensive and not available in every clinical setting, may be unsuitable for clinical use. Furthermore, measuring proprioceptive accuracy in the elbow joint only does not provide sufficient information to therapists for development of a treatment plan. Future studies are necessary to establish standardized testing procedures and to verify the reliability and validity of the arm position and motion matching tests for evidence-based practice in clinics.

\section{Clinical implications}

During movement planning and execution, proprioception that provides movement and position information of needed segments is essential to the CNS for taking into account all body segments involved in the desired task. ${ }^{52}$ An adequate assessment of proprioceptive and kinesthetic accuracy can provide further insights into implementing a treatment plan. After proper training, clinical practitioners can gather preliminary information on an individual's accuracy of position and motion sense by manually performing the test, which is inexpensive and easily administered in a clinical setting. We hope that the results of the current study will add more evidence to enhance evidence-based practice.

\section{Limitations}

There are several limitations to our study. First, the ratio of men to women was uneven and this may have influenced results. Although there is no clear evidence to suggest that sex has an effect on proprioceptive accuracy, it should be investigated in future studies. Second, we applied testing protocols only to healthy adults. Potential applications for patients with neurological disorders should be further explored. Third, most joint angles were tested in the sagittal plane (the exception was shoulder abduction). Determining whether horizontal plane motion can generate similar findings requires further investigation. Finally, we only recruited right-handed subjects. Previous studies have reported that left-handed individuals do not display uniform brain activation patterns during execution of movement and processing of language. ${ }^{53,54}$ Determining the effect of handedness on arm matching position and motion tests warrants further examination.

\section{Acknowledgments}

This project was supported in part by grants from the National Science Council of Taiwan (NSC100-2410-H-182015-MY2 and NSC102-2314-B-182-009-MY3) and the Healthy Aging Research Center at Chang Gung University (EMRPD1D0291, CMRPD1B0331).

\section{Disclosure}

The authors report no conflicts of interest in this work.

\section{References}

1. Sherrington CS. On the proprio-ceptive system, especially in its reflex aspect. Brain. 1907;29:467-482.

2. Gentilucci M, Negrotti A. Dissociation between perception and visuomotor transformation during reproduction of remembered distances. J Neurophysiol. 1994;72(4):2026-2030.

3. Gentilucci M, Toni I, Chieffi S, Pavesi G. The role of proprioception in the control of prehension movements: a kinematic study in a peripherally deafferented patient and in normal subjects. Exp Brain Res. 1994;99(3):483-500.

4. Sainburg RL, Ghilardi MF, Poizner H, Ghez C. Control of limb dynamics in normal subjects and patients without proprioception. J Neurophysiol. 1995;73(2):820-835.

5. Sainburg RL, Poizner H, Ghez C. Loss of proprioception produces deficits in interjoint coordination. J Neurophysiol. 1993;70(5):2136-2147.

6. Bentzel K. Assessing abilities and capacities: sensation. In: Radomski MV, Trombly LCA, Latham CAT, editors. Occupational therapy for physical dysfunction. 6th ed. Baltimore, Maryland: Lippincott Williams \& Wilkins; 2008:212-233.

7. Lincoln NB, Crow JL, Jackson JM, Waters GR, Adams SA, Hodgson P. The unreliability of sensory assessments. Clinical Rehabilitation. 1991;(5):273-282.

8. Juul-Kristensen B, Lund H, Hansen K, Christensen H, DanneskioldSamsoe B, Bliddal H. Test-retest reliability of joint position and kinesthetic sense in the elbow of healthy subjects. Physiother Theory Pract. 2008;24(1):65-72.

9. Goble DJ. Proprioceptive acuity assessment via joint position matching: from basic science to general practice. Phys Ther. 2010;90(8):1176-1184.

10. Goble DJ, Coxon JP, Wenderoth N, Van Impe A, Swinnen SP. Proprioceptive sensibility in the elderly: degeneration, functional consequences and plastic-adaptive processes. Neurosci Biobehav Rev. 2009;33(3): 271-278.

11. O'Suilleabhain P, Bullard J, Dewey RB. Proprioception in Parkinson's disease is acutely depressed by dopaminergic medications. J Neurol Neurosurg Psychiatry. 2001;71(5):607-610.

12. Adamo DE, Alexander NB, Brown SH. The influence of age and physical activity on upper limb proprioceptive ability. J Aging Phys Act. 2009;17(3):272-293.

13. Kaplan FS, Nixon JE, Reitz M, Rindfleish L, Tucker J. Age-related changes in proprioception and sensation of joint position. Acta Orthop Scand. 1985;56(1):72-74.

14. Adamo DE, Martin BJ, Brown SH. Age-related differences in upper limb proprioceptive acuity. Percept Mot Skills. 2007;104(3 Pt 2): 1297-1309.

15. Barrack RL, Skinner HB, Cook SD, Haddad RJ Jr. Effect of articular disease and total knee arthroplasty on knee joint-position sense. J Neurophysiol. 1983;50(3):684-687. 
16. Konczak J, Li KY, Tuite PJ, Poizner H. Haptic perception of object curvature in Parkinson's disease. PLoS One. 2008;3(7):e2625.

17. Maschke M, Gomez CM, Tuite PJ, Konczak J. Dysfunction of the basal ganglia, but not the cerebellum, impairs kinaesthesia. Brain 2003;126(Pt 10):2312-2322.

18. Putzki N, Stude P, Konczak J, Graf K, Diener HC, Maschke M. Kinesthesia is impaired in focal dystonia. Mov Disord. 2006;21(6):754-760.

19. Dukelow SP, Herter TM, Moore KD, et al. Quantitative assessment of limb position sense following stroke. Neurorehabil Neural Repair. 2010;24(2):178-187.

20. Carey LM, Oke LE, Matyas TA. Impaired limb position sense after stroke: a quantitative test for clinical use. Arch Phys Med Rehabil. 1996;77(12):1271-1278.

21. Sullivan JE, Hedman LD. Sensory dysfunction following stroke: incidence, significance, examination, and intervention. Top Stroke Rehabil. 2008;15(3):200-217.

22. Semrau JA, Herter TM, Scott SH, Dukelow SP. Robotic identification of kinesthetic deficits after stroke. Stroke. 2013;44(12):3414-3421.

23. Oldfield RC. The assessment and analysis of handedness: the Edinburgh inventory. Neuropsychologia. 1971;9(1):97-113.

24. Folstein MF, Folstein SE, McHugh PR. "Mini-mental state". A practical method for grading the cognitive state of patients for the clinician. J Psychiatr Res. 1975;12(3):189-198.

25. Alt Murphy M, Sunnerhagen KS, Johnels B, Willen C. Three-dimensional kinematic motion analysis of a daily activity drinking from a glass: a pilot study. J Neuroeng Rehabil. 2006;3:18.

26. Cirstea MC, Levin MF. Compensatory strategies for reaching in stroke. Brain. 2000;123(Pt 5):940-953.

27. Michaelsen SM, Luta A, Roby-Brami A, Levin MF. Effect of trunk restraint on the recovery of reaching movements in hemiparetic patients. Stroke. 2001;32(8):1875-1883.

28. Hall LA, McCloskey DI. Detections of movements imposed on finger, elbow and shoulder joints. J Physiol. 1983;335:519-533.

29. Goble DJ, Brown SH. Upper limb asymmetries in the matching of proprioceptive versus visual targets. J Neurophysiol. 2008;99(6): 3063-3074.

30. Goble DJ, Lewis CA, Hurvitz EA, Brown SH. Development of upper limb proprioceptive accuracy in children and adolescents. Hum Mov Sci. 2005;24(2):155-170.

31. Westlake KP, Culham EG. Sensory-specific balance training in older adults: effect on proprioceptive reintegration and cognitive demands. Phys Ther. 2007;87(10):1274-1283.

32. Chinn S. Statistics in respiratory medicine. 2. Repeatability and method comparison. Thorax. 1991;46(6):454-456.

33. Allen TJ, Proske U. Effect of muscle fatigue on the sense of limb position and movement. Exp Brain Res. 2006;170(1):30-38.

34. Kerr GK, Worringham CJ. Velocity perception and proprioception. Adv Exp Med Biol. 2002;508:79-86.

35. Hagura N, Oouchida Y, Aramaki Y, et al. Visuokinesthetic perception of hand movement is mediated by cerebro-cerebellar interaction between the left cerebellum and right parietal cortex. Cereb Cortex. 2009;19(1): 176-186.

36. Naito E, Roland PE, Grefkes C, et al. Dominance of the right hemisphere and role of area 2 in human kinesthesia. $J$ Neurophysiol. 2005;93(2):1020-1034.
37. Proske U, Gandevia SC. The kinaesthetic senses. J Physiol. 2009; 587(Pt 17):4139-4146.

38. Brown LE, Rosenbaum DA, Sainburg RL. Limb position drift: implications for control of posture and movement. J Neurophysiol. 2003;90(5):3105-3118.

39. Scott SH, Loeb GE. The computation of position sense from spindles in mono- and multiarticular muscles. J Neurosci. 1994;14(12): 7529-7540.

40. Goble DJ, Brown SH. Task-dependent asymmetries in the utilization of proprioceptive feedback for goal-directed movement. Exp Brain Res. 2007;180(4):693-704

41. Goble DJ, Brown SH. Dynamic proprioceptive target-matching behavior in the upper limb: effects of speed, task difficulty and arm/hemisphere asymmetries. Behav Brain Res. 2009;200(1):7-14.

42. Riolo-Quinn L. Relationship of hand preference to accuracy on a thumbpositioning task. Percept Mot Skills. 1991;73(1):267-273.

43. Gandevia SC, Smith JL, Crawford M, Proske U, Taylor JL. Motor commands contribute to human position sense. J Physiol. 2006; 571(Pt 3):703-710.

44. Allen TJ, Ansems GE, Proske U. Effects of muscle conditioning on position sense at the human forearm during loading or fatigue of elbow flexors and the role of the sense of effort. $J$ Physiol. 2007;580(Pt 2): 423-434.

45. Winter JA, Allen TJ, Proske U. Muscle spindle signals combine with the sense of effort to indicate limb position. J Physiol. 2005;568(Pt 3): $1035-1046$.

46. Gritsenko V, Krouchev NI, Kalaska JF. Afferent input, efference copy, signal noise, and biases in perception of joint angle during active versus passive elbow movements. $J$ Neurophysiol. 2007;98(3): $1140-1154$.

47. Ansems GE, Allen TJ, Proske U. Position sense at the human forearm in the horizontal plane during loading and vibration of elbow muscles. J Physiol. 2006;576(Pt 2):445-455.

48. Itaguchi Y, Fukuzawa K. Effects of arm stiffness and muscle effort on position reproduction error in the horizontal plane. Percept Mot Skills. 2012;114(3):757-773.

49. Itaguchi Y, Fukuzawa K. The influence of the indicator arm on end point distribution in proprioceptive localization with multi-joint arms Exp Brain Res. 2012;222(1-2):77-88.

50. Lackner JR, DiZio PA. Aspects of body self-calibration. Trends Cogn Sci. 2000;4(7):279-288.

51. Winward CE, Halligan PW, Wade DT. Current practice and clinical relevance of somatosensory assessment after stroke. Clin Rehabil. 1999; 13(1):48-55.

52. Hasan Z, Stuart DG. Animal solutions to problems of movement control: the role of proprioceptors. Annu Rev Neurosci. 1988;11: 199-223.

53. Pujol J, Deus J, Losilla JM, Capdevila A. Cerebral lateralization of language in normal left-handed people studied by functional MRI Neurology. 1999;52(5):1038-1043.

54. Verstynen T, Diedrichsen J, Albert N, Aparicio P, Ivry RB. Ipsilateral motor cortex activity during unimanual hand movements relates to task complexity. J Neurophysiol. 2005;93(3):1209-1222.
Clinical Interventions in Aging

\section{Publish your work in this journal}

Clinical Interventions in Aging is an international, peer-reviewed journal focusing on evidence-based reports on the value or lack thereof of treatments intended to prevent or delay the onset of maladaptive correlates of aging in human beings. This journal is indexed on PubMed Central, MedLine,

\section{Dovepress}

CAS, Scopus and the Elsevier Bibliographic databases. The manuscript management system is completely online and includes a very quick and fair peer-review system, which is all easy to use. Visit http://www.dovepress. com/testimonials.php to read real quotes from published authors. 